\title{
Leadershipstile im Kontext von Schulentwicklungsprozessen
}

\author{
Entwicklung und Validierung eines Inventarmoduls unter Kombination einer \\ domänenspezifischen mit einer funktionsanalytischen Perspektive
}

\author{
Franz Hofmann ${ }^{1} \cdot$ Matteo Carmignola $^{1}$ (D)
}

Received: 17 February 2021 / Accepted: 7 May 2021 / Published online: 31 May 2021

(c) The Author(s) 2021

\begin{abstract}
The focus on educational quality on the single school-level and the aim for ongoing school-improvement direct the attention to investigating the quality of leadership of school administrators. However, despite a large number of educational leadership models, some do not provide a sound theoretical framework, as they confound domains and tasks with the leadership style or identify leadership styles that are based on inconsistent assumptions of motivational psychology. In this paper, a leadership model for the specific context of leading school improvement processes is designed using a functional approach, which is based on the theory of personality systems interactions (PSI). We identified four styles of leadership enactment: the (1) visionary-enthusiastic, the (2) (latent) anxious-goal-fixated, the (3) passive-avoidant, and the (4) self-directed style. The newly developed instrument was validated for a sample of $n=901$ teachers and $k=75$ principals. In the discussion section, particular attention is paid to the self-directed style, for which favorable effects can be postulated both in the change management of school improvement processes and in regard to the motivational experience of teachers.
\end{abstract}

Keywords Educational leadership $\cdot$ Leadership style $\cdot$ School improvement planning $\cdot$ Change-management $\cdot$ PSI-theory

\section{Zusammenfassung}

Durch den verstärkten Fokus auf die Ebene von Einzelschulen in der Qualitätssicherung und Weiterentwicklung des Bildungsangebotes wird ein besonderes Augenmerk auch auf die Führungsqualität von Schulleitungen gerichtet, für die das Leitungshandeln in Schulen bisher nur unzureichend valide operationalisiert wurde. So konfundieren derzeit vorliegende Modelle Domänen und Aufgabenbereiche mit dem Stil des Führungshandelns oder formulieren kontextgelöst Leadershipstile, die auf einer teils inkonsistenten motivationspsychologischen Grundlage basieren. In diesem Beitrag wird unter Einbezug der Funktionsanalyse im Sinne der PSI-Theorie ein Leadershipmodell für den spezifischen Kontext der Leitung von Schulentwicklungsprozessen entworfen und an einer Stichprobe von $n=901$ Lehrpersonen und $k=75$ Schulleitungen validiert. Aus einer funktionsanalytischen Perspektive werden dabei vier Leitungsstile modelliert: der (1) visionär-begeisternde, der (2) (latent) ängstlich-zielfixierte, der (3) passiv-vermeidende und der (4) selbstgesteuerte Leadershipstil. In der Diskussion wird die Aufmerksamkeit insbesondere auf den selbstgesteuerten Stil gerichtet, für den günstige Effekte sowohl im ChangeManagement von Schulentwicklungsprozessen postuliert und auch in der vorliegenden Konstruktvalidierung in Bezug auf das motivationale Erleben von Lehrpersonen nachgewiesen werden können.

Schlüsselwörter Schulleitungshandeln · Leadershipstil $\cdot$ Schulentwicklung $\cdot$ Change-management · PSI-Theorie

Franz Hofmann

franz.hofmann@sbg.ac.at

1 School of Education, Universität Salzburg, Salzburg, Österreich

\section{Schulleitungshandeln im Fokus der Bildungsforschung}

Menschen und Organisationen zu leiten gehört zur ureigensten pädagogischen Aufgabe, die nicht zuletzt auch etymologisch durch das griechische Verb ăyeıv (agein: führen, leiten; im 
Substantiv $\pi \alpha \_\delta \alpha \gamma \omega \gamma o ́ \varsigma$ paidagogós: Pädagoge, Erzieher) grundgelegt wird. Während die Figur der Schulleitungsperson, welche regional oder schulformspezifisch in der Bezeichnung zwischen Schuldirektor/in, Schulführungskraft oder Schulmanager/in variieren kann, keineswegs neu, sondern mit der Geschichte des Schulwesens verwoben ist (Bonsen 2016; Göhlich 2018), unterliegen die Anforderungen an Schulleitungskräfte und deren Rollenprofil dem bildungspolitischen Wandel (Schratz et al. 2016; Schwanenberg et al. 2020).

Für den aktuellen Bildungsdiskurs erscheinen dabei zwei Entwicklungen besonders wesentlich, die in den Begriffen des New Public Management (Pashiardis und Brauckmann 2019) und der ergebnisorientierten Steuerung des Bildungssystems (Altrichter und Maag Merki 2016; Demski 2017) verortet werden können und insbesondere die Verantwortung der Schulleitung für Lernoutcomes als ein zentrales Qualitätsmerkmal des Bildungsprozesses in den Fokus stellen. Dieses Postulat wird durch einen stetig wachsenden Literaturkorpus an empirischer Forschungsliteratur gestützt, in dem praktisch relevante Effekte von Schulleitungshandeln auf Schüler/innenoutcomes (Leithwood et al. 2010; Leithwood und Jantzi 2006) berichtet werden, neben günstigen Outcomes bei Lehrpersonen etwa hinsichtlich deren Motivation und Arbeitszufriedenheit (Eyal und Roth 2011; Kovjanic et al. 2013; Nguni et al. 2006).

Um die beobachteten Effekte auf Merkmale der Führungsperson oder auf Charakteristika des Leitungshandelns zurückzuführen und theoretisch zu beleuchten, wurden für den Schulkontext spezifische Modelle entworfen oder etablierte Führungsmodelle aus der betriebswirtschaftlichen Anwendungsforschung (in Ansätzen) für den Bildungskontext adaptiert (Leithwood und Jantzi 2005). Wie in diesem Beitrag an zwei Leadershipmodellen aufgezeigt werden soll, erfüllen gängige Instrumentarien den Anspruch in der Regel nicht, sowohl kontextspezifische Handlungsdimensionen als auch übergreifende Leadershipstile abzubilden, was unter anderem auch die Konstruktvalidität der Instrumentarien mindert.

In diesem Beitrag soll daher auf Basis der PersönlichkeitsSystem-Interaktionen-Theorie (PSI; Kuhl 2001, 2010) eine erste Facette eines multidimensionalen Leadershipinventars validiert werden. Mit besonderer Berücksichtigung des Führungshandelns im Kontext der Schulentwicklungsarbeit werden dabei aus funktionsanalytischer Perspektive vier Leadershipstile abgeleitet, um eine persönlichkeitspsychologisch differenzierte Sichtweise auf das Schulleitungshandeln zu konzipieren.

\section{Orientierung an Domänen oder Umsetzungsformen?}

Das Streben nach einer verstärkten Professionalisierung und Qualifizierung von Schulleitungspersonen (Bonsen 2016; Brauckmann und Böse 2018) erfordert eine gleichermaßen bildungswissenschaftlich wie persönlichkeitspsychologisch informierte theoretische Fundierung für Grundlagen- und Begleitforschung zum Schulleitungsberuf sowie zur Erstellung von Curricula für Qualifizierungslehrgänge. Während etwa Kompetenzmodelle teilweise auch spezifisch für Qualifizierungslehrgänge entworfen werden (Schratz et al. 2016, S. 237), können nach Gumus et al. (2018) etwa ein Dutzend Leadershiptheorien aufgelistet werden, welche in Large-Scale-Untersuchungen implementiert wurden. Darunter finden sich die Stile der transformational und transactional leadership, welche dem Full-Range-Model nach Bass und Avolio (1995b) entnommen wurden, ebenso wie die Leadershipdimensionen der instructional leadership (Hallinger und Murphy 1985) oder der distributed/collaborative leadership (Mayrowetz 2008), die etwa auch im "PashiardisBrauckmann Holistic Leadership Framework" (Brauckmann und Pashiardis 2011) als einzelne "styles" zu einem Gesamtmodell zusammengefügt werden.

Analysiert man Leadershipmodelle aus der Perspektive der konzeptuellen bzw. theoretischen Fundierung, finden sich in diesen zwei exemplarisch hervorgehobenen Makromodellen zwei grundlegende Stile von Leadership, die sich voneinander abgrenzen lassen:

\subsection{Transformationale vs. transaktionale Leadership_das Full-Range-Model nach Bass und Avolio}

Das Full-Range-Leadershipmodell von Bass und Avolio (1995b) unterscheidet drei Grunddimensionen von Leadership: (1) die transformationale, (2) die transaktionale und (3) die Laissez-faire-Führung. Transformationale Führung ist dadurch charakterisiert, dass Führungspersonen ihren Idealen und Überzeugungen entsprechend andere dafür gewinnen wollen, gewünschte Handlungen in Gang zu setzen oder Mitarbeiter/innen - sofern es sich dabei nicht um Prozessschritte handelt, die bereits im Detail festgelegt sind - Handlungsfreiräume gewähren, sich auf individuelle Art und Weise in ihrer Arbeit einbringen zu können (Bass und Avolio 1994). Beim transaktionalen Stil geht es darum, dass Führungskräfte bestimmte Aufgaben anordnen, die Qualität der Realisierung dieser einzelnen Aufgaben kontrollieren und ggf. mit Belohnungen agieren. Kennzeichnend ist dabei gerade auch die Orientierung an Fehlern und Abweichungen oder die Vermeidung von negativen Entwicklungen (Avolio und Bass 2002).

Ausgehend von Untersuchungen aus der Wahlkampfpolitik durch Burns (1978) wurden schrittweise die Führungsstile ausdifferenziert (Avolio und Bass 2002); das Full-RangeModel bildet seit den 80er Jahren des letzten Jahrtausends eines der am häufigsten untersuchten Rahmenmodelle von 
Leadership in Organisationen. Daher werden - mit Blick auch auf den Schulkontext - hierbei gerade Stile ohne Bezug auf inhaltliche Domänen operationalisiert. Zuweilen wird das Full-Range-Model auch als ein Kontinuum dargestellt, in dem auf einer vertikalen Achse von ineffektiv zu effektiv und einer horizontalen Achse von passiv zu aktiv, acht Substile verortet werden (Bass und Avolio 1995b; Furtner und Baldegger 2013): Am Extrem "ineffektiv und passiv" steht der Stil der Laissez-faire-Führung, gefolgt von den Stilen der transaktionalen Führung (management by exception passive und active) und der vier Stile der transformationalen Führung; hierbei wird dem Substil "idealized behavior" die höchste Ausprägung an Aktivität und Effektivität zugeschrieben, wenn auch keine umfassende empirische Befundlage zu dieser Abstufung hinsichtlich Effektivität und Outcomes angeführt wird. Passender erscheint den Autoren des vorliegenden Beitrags die Bezeichnung der Vertikalachse mit den Polaritäten "hoch positiv affektiv "und" hoch negativ affektiv", was etwa auch durch die inhaltliche Nähe der transformationalen Führung zur charismatischen Führung zum Ausdruck kommt.

Für den Bildungsbereich konnten für die Stile der transformationalen Führung positive Zusammenhänge zur Motivationsqualität von Lehrpersonen (Eyal und Roth 2011; Leithwood und Jantzi 2006), zu positivem Schulklima (McCarley et al. 2016), zu kollektiven Selbstwirksamkeitserwartungen bei Lehrpersonen (Ninković und Knežević Florić 2018) sowie höhere Werte an Arbeitszufriedenheit (Bogler 2001; Griffith 2004) nachgewiesen werden. Für die transaktionale Führung liegen Befunde über höhere Ausprägungen von Formen der kontrollierten Motivationsregulation sowie von direkten und über die Motivationsqualität mediierten Effekten auf die emotionale Erschöpfung (Eyal und Roth 2011) sowie zur Berufszufriedenheit vor (Bogler 2001). Insbesondere werden diese negativen Effekte über den Stil des management by exception passive berichtet (Nguni et al. 2006), während für das management by exception active meist keine gerichteten bzw. signifikanten Zusammenhänge mit Lehrer/innenoutcomes erzielt werden.

\subsection{Domänen- und kontextspezifisches Schulleitungshandeln}

Im Gegensatz zum Full-Range-Model steht beispielsweise das Pashiardis-Brauckmann Holistic Leadership Framework: Brauckmann und Pashiardis (2011) führen dabei fünf Stile (styles) auf: (1) instructional, (2) structuring, (3) participative, (4) entrepreneurial und (5) personnel development. Diese fünf Konstrukte werden auf der Grundlage eines systematischen Literaturreviews und zum Teil durch bereits existierende Instrumentarien in einer Validierungsstudie als Leadershipmodell für Schulleitungen mit einem
Mittelwertsvergleich zwischen sieben europäischen Schulsystemen präsentiert.

Bei einer näheren Betrachtung wird ersichtlich, dass bei dessen Dimensionierung eine Mischung zwischen Inhaltsbereichen und Führungsstilen zu konstatieren ist. Im deutschen wie im englischen Sprachgebrauch wird Stil als "Art und Weise der Ausführung" (Wahrig 2000) bzw. style als "a manner or way of doing something" (Cambridge Dictionary 2014) definiert, sodass - vom Begriffsverständnis her betrachtet - der Fokus nicht auf dem Tätigkeitsfeld liegt, sondern impliziert wird, dass ein und dieselbe Tätigkeit bzw. Aufgabe auf unterschiedliche Art und Weise ausgeführt werden kann. Demgegenüber folgen die Autoren des Pashiardis-Brauckmann Holistic Leadership Framework einer eigenen Arbeitsdefinition, welche einen leadership style als "the nexus of all those behaviours and practices that school principals use in order to influence the behaviours of others" (Brauckmann und Pashiardis 2011, S. 13) auffasst. Ein "Style" bezeichnet demnach gleichermaßen die Fokussierung auf eine bestimmte Domäne (z. B. Unterrichts- oder Personalentwicklung) und die Art und Weise des kommunikativen Agierens. In der Operationalisierung und Modellierung der Faktoren im Holistic Leadership Framework (Brauckmann und Pashiardis 2011) werden diese komplementären Dimensionen nicht weiter unterschieden. In analoger Weise trifft das etwa auch für das LEAD-Führungsfeedback zu: Der Kompetenzbereich "Strategieorientierung" enthält einzelne für Unternehmensführung spezifische Aufgabenfelder (z. B. Marktchancen erkennen) genauso wie eher stiltypische Kompetenzbereiche (z. B. Verantwortungsübertragung oder Perspektivenwechsel im Kontext der Mitarbeiter/innenentwicklung; vgl. Schmidt-Huber et al. 2014; vgl. diese Vermischung auch bei Brauckmann und Eder 2019, S. 9).

\subsection{Anliegen in der Weiterentwicklung}

An den oben angeführten Leadershipmodellen wird exemplarisch ersichtlich, dass weiterhin Bedarf nach einem umfassenden Modell besteht, das sowohl spezifisch die Aufgaben und Domänen des Schulleitungshandelns als auch eine solide differentialpsychologische Grundlage aufweisen kann. In dieser Studie wird deshalb in einem ersten Schritt eine Systematik der berufsspezifischen Handlungsdomänen von Schulleitungen entworfen (vgl. Tabelle 1). In weiterer Folge werden am Beispiel der Dimension D3_org, in der die Initiierung und Implementation von Schulentwicklungsprozessen abgebildet wird, vier verschiedene Leadershipstile formuliert, welche je persönlichkeitspsychologisch begründete, unterschiedliche Umsetzungsformen umfassen. 
Tabelle $13 \times 2$ Feldermatrix für Aktivitäten von Schulführungskräften

\begin{tabular}{|c|c|c|}
\hline Dimensionen & Makroebene: organisational & Mikroebene: personal \\
\hline $\begin{array}{l}\text { D1: ordnen } \\
\text { Strukturen implementieren und für deren } \\
\text { Wirksamkeit sorgen: Begrenzungs- } \\
\text { maßnahmen }\end{array}$ & $\begin{array}{l}\text { D1_org: Organisationsstrukturen funktional zu } \\
\text { den Zielen machen } \\
\text { (Maßnahmen zur Institutionalisierung z. B. von } \\
\text { Innovationen) }\end{array}$ & $\begin{array}{l}\text { D1_pers: Regeln, Normen mit Blick auf } \\
\text { pädagogische Ideale der Schule (Interak- } \\
\text { tionsebenen hierarchisch und symmetrisch: } \\
\text { SFK, Lehrpersonen, Schüler/innen, Eltern, } \\
\text { Verwaltungspersonal) }\end{array}$ \\
\hline $\begin{array}{l}\text { D2: vernetzen } \\
\text { für Vernetzung sorgen }\end{array}$ & $\begin{array}{l}\text { D2_org: Vernetzung der Schule mit externen } \\
\text { Instanzen (Eltern, Partnerschulen, öffentliche } \\
\text { Einrichtungen, Wirtschaft etc.) }\end{array}$ & D2_pers: Kooperation zwischen Lehrpersonen \\
\hline $\begin{array}{l}\text { D3: Entwicklung stimulieren } \\
\text { zone of proximal development }\end{array}$ & $\begin{array}{l}\text { D3_org: Maßnahmen zur Organisations- (change } \\
\text { management) und Unterrichtsentwicklung }\end{array}$ & $\begin{array}{l}\text { D3_pers: Maßnahmen zur Personalentwick- } \\
\text { lung, betrachtet unter der Perspektive der } \\
\text { Fähigkeit und Bereitschaft zur } \\
\text { Selbstentwicklung }\end{array}$ \\
\hline
\end{tabular}

\section{Konzeptuelle sowie theoretische Fundierung eines Leadershipinventars für Schulleitungen}

\subsection{Systematik von operativen Domänen des Schulleitungshandelns}

Bei unserer Entwicklungsarbeit eines Inventars für Schulführungskräfte generieren wir eine Struktur, die zwei Dimensionen miteinander verknüpft (vgl. Tabelle 1): eine aktivitätsorientierte Dimension, in der davon ausgegangen wird, dass Schulführungskräfte in ihrer beruflichen Tätigkeit ordnend, vernetzend und entwicklungsstimulierend tätig sind und eine adressat/innenorientierte Dimension, weil sich diese Aktivitäten sowohl auf die Schule als organisationales Gebilde als auch auf die schulischen Bezugsgruppen (Schüler/innen, Lehrer/innen, Eltern, Verwaltungspersonal etc.; personale Ebene) beziehen können: "Ordnen" bedeutet in diesem Zusammenhang, dass sie Strukturen implementieren, die zu den Profilen und Leitbildern einer Schule funktional sind, und den in einer Organisation arbeitenden Menschen mit einem solchen Rahmen Orientierung ( $\mathrm{z}$. B. Interaktionskultur) und Sicherheit bieten. "Vernetzen" bedeutet gerade für eine eher ins Zelluläre driftende Organisation wie die Schule, dass Schulführungskräfte Schulkooperationen mit anderen Schulen bzw. Institutionen anstoßen (organisationale Ebene) und gleichermaßen die empirisch betrachtet nicht einfache Aufgabe wahrnehmen, die schulischen Bezugsgruppen in eine der Bildungsqualität dienliche Kooperation zu bringen. Aufgrund der durch die Einführung von Schulautonomie bedingten Tatsache, dass Schulen als dynamische, entwicklungsfähige Organisationen verstanden werden, hat die Schulführungskraft neben der früher als zentral angesehenen Aufgabe der Schulverwaltung auch die Pflicht, für die Entwicklung einer funktionalen und zeitgemäßen Schulstruktur und des in ihr arbeitenden Personals zu sorgen. Es ergibt sich daher für eine umfassende Darstellung des breiten Aufgabenbereichs einer Schulführungskraft auf einer mittleren Abstraktionsebene eine $3 \times 2$ Feldermatrix (vgl. Tabelle 1), in der sich auch die in der aktuellen Schulentwicklungsdiskussion drei unterschiedenen Aufgaben der Organisations-, Unterrichts- und Personalentwicklung verorten lassen.

Im vorliegenden Ansatz wird im Unterschied zu den oben referierten Modellen einerseits zwischen der Frage differenziert, welche berufsspezifischen operativen Domänen für die Arbeit einer Schulführungskraft voneinander abzugrenzen sind (vgl. Tabelle 1) und andererseits, in welcher Art und Weise eine Schulführungskraft jeweils bei der Bewältigung der in den einzelnen Domänen anfallenden Aufgaben agiert (vgl. die Leadershipstile in Tabelle 2).

\subsection{Funktionsanalytisch definierte Umsetzungsformen als Stile des Leitungshandelns}

Im Hinblick auf die Umsetzung der anfallenden Aufgaben grenzen wir vier Leadershipstile voneinander ab, die auf der Basis zentraler Thesen der Persönlichkeits-System-Interaktionen-Theorie nach Kuhl $(2001,2010)$ konzipiert sind. In der PSI-Theorie geht man davon aus, dass Handeln bzw. Handlungsbahnung und Selbsterleben insbesondere durch vier kognitive Makrosysteme und deren Interaktionen generiert werden, wodurch sich diagnostisch die Möglichkeit ergibt, Handeln und Selbsterleben funktionsanalytisch zu erklären. Da jedes dieser vier Makrosysteme mit einer bestimmten Affektlage assoziiert ist, kann Selbststeuerung auch als elaborierte Fähigkeit der individuellen Affektregulation betrachtet werden.

Das Zusammenspiel der vier psychischen Makrosysteme (vgl. Abbildung 1) wird durch zwei Affektsysteme moduliert. Je nach veranlagter Sensibilität (auch Primärpersönlichkeit genannt; Kuhl 2013, S. 13) für den positiven $(\mathrm{A}+\mathrm{I}$ Freude, Begeisterung) oder negativen Affekt (A-I Empfindsamkeit, Bestrafungsangst) sind Selbsterleben 
Tabelle 2 Überblick über die vier Leitungsstile, deren Affektgeladenheit (vgl. Kuhl 2013) sowie deren zentrale Merkmale

\begin{tabular}{|c|c|c|}
\hline Bezeichnung & Affektkonfiguration & Merkmale \\
\hline Visionär-begeisternder Stil & $\begin{array}{l}\text { Positiver Affekt; } \\
\text { unter Beteiligung des Selbstsystems; } \\
A+/ A(-)\end{array}$ & $\begin{array}{l}\text { Begeisternd sein; mit einer Idee vorangehen; im } \\
\text { Außen Anerkennung finden wollen; andere über- } \\
\text { zeugen und gewinnen; Bedenken und Widerstand } \\
\text { übersehen }\end{array}$ \\
\hline (Latent) ängstlich-zielfixierter Stil & $\begin{array}{l}\text { Negativer Affekt; } \\
\text { A- }\end{array}$ & $\begin{array}{l}\text { Das Ziel unbedingt erreichen müssen; das Scheitern } \\
\text { hintanhalten wollen; "Augen zu und durch"-Mental- } \\
\text { ität; anderen etwas vorschreiben und sie kontrol- } \\
\text { lieren }\end{array}$ \\
\hline Passiv-vermeidender Stil & $\begin{array}{l}\text { Negativer und gedämpfter positiver Affekt; } \\
\mathrm{A}-/ \mathrm{A}(+)\end{array}$ & $\begin{array}{l}\text { Sich von der Widersprüchlichkeit der Bedürfnisse und } \\
\text { Wünsche in einem Entwicklungsprozess lähmen } \\
\text { lassen; nichts mehr tun mit Verweis auf bisherige } \\
\text { Ineffektivität; die Selbstzuversicht auf wirksames } \\
\text { Machthandeln verloren haben }\end{array}$ \\
\hline Selbstgesteuerter Stil & $\begin{array}{l}\text { In der Affektkonfiguration nur situational } \\
\text { bestimmbar; jedenfalls unter Beteiligung des } \\
\text { Erfahrungsgedächtnisses und des Selbstsystems; } \\
\text { A(-) }\end{array}$ & $\begin{array}{l}\text { Das eigene Ideal oder die eigene Entwicklungsidee } \\
\text { wertschätzen bei gleichzeitiger Ernstnahme der } \\
\text { Bedenken anderer dagegen; die Entwicklungsidee } \\
\text { gemeinsam mit anderen generieren; diese Idee } \\
\text { während der Umsetzung ggf. adaptieren (gegen eine } \\
\text { "Alles oder nichts"-Mentalität); den "kairos" für die } \\
\text { Realisierung eigener Ideen nutzen können }\end{array}$ \\
\hline
\end{tabular}

Abbildung 1 Vereinfachte Darstellung der Makrosysteme der PSI-Theorie und der zugeordneten Affektsysteme (vgl. Kuhl und Alsleben 2012, S. 139; Kuhl et al. 2010, S. 47)
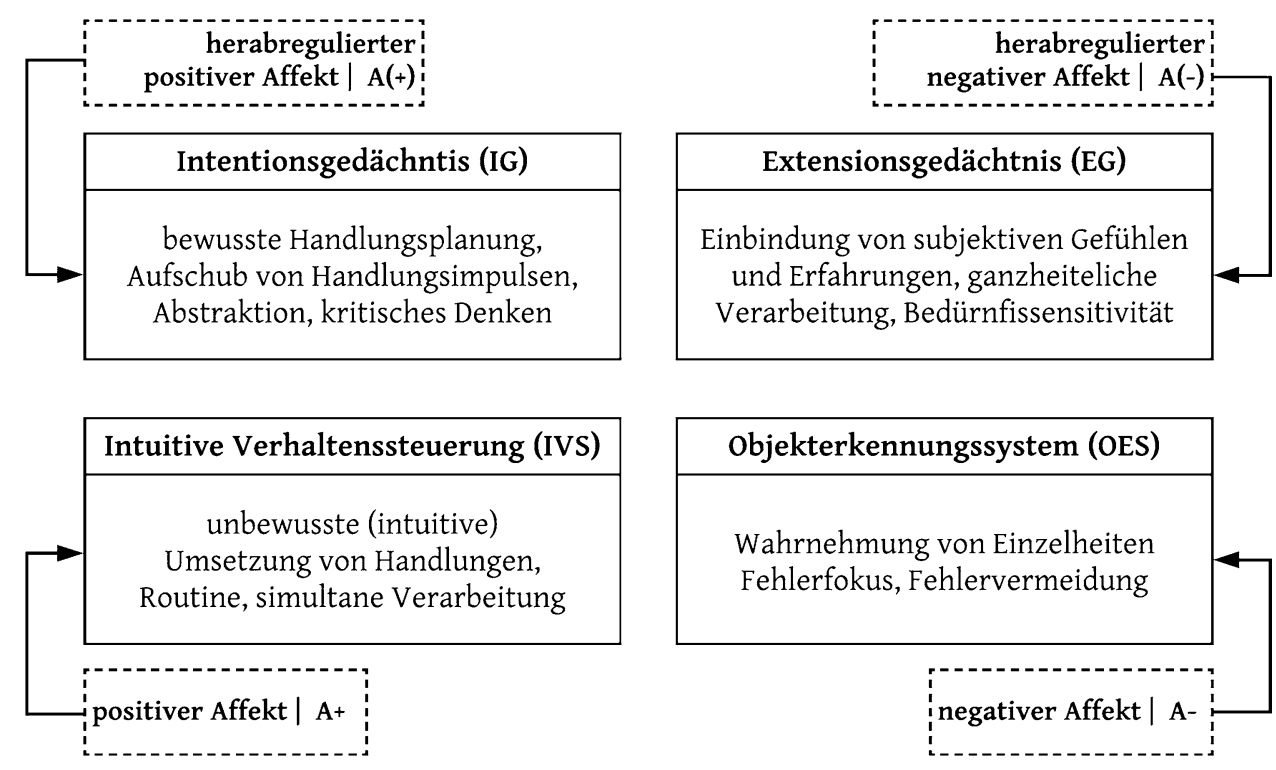

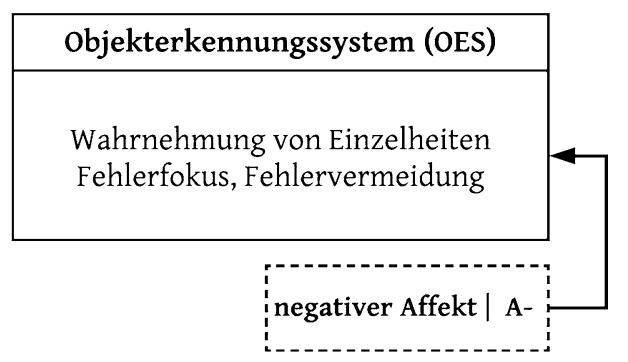

und Handlungsbahnung stärker mit einem Makrosystem verknüpft: Die Intuitive Verhaltenssteuerung (IVS) wird durch den positiven Affekt aktiviert, der energetisierend wirkt und routinemäßige Handlungen bahnt bzw. erleichtert (Kuhl 2010, S. 84). Der negative Affekt ist hingegen mit der Funktion der Objekterkennung (OES) assoziiert, welche in der Wahrnehmung den Fokus auf Unstimmigkeiten, Fehler sowie isolierte Einzelelemente lenkt (Kuhl 2001, 161f).

Neben einer Aktivierung durch eine hohe Affektausprägung kann auch durch die Herabregulation des positiven [A(+) | Nüchternheit, Lustlosigkeit) und des negativen Affektes $\left[\mathrm{A}(-) \mid\right.$ Robustheit, Gelassenheit ${ }^{1}$ eine Aktivierung von kognitiven Makrosystemen erfolgen: Die Dämpfung des positiven Affektes $[\mathrm{A}(+)]$ aktiviert die Funktion des Intentionsgedächtnisses (IG), bei dem eine Handlungsumsetzung gebremst wird, um so eine sequentielle Planung von logischen Handlungsschritten zu entwerfen (Kuhl et al. 2010, S. 47). Die Herabregulation des negativen Affektes [A(-)] ermöglicht indes den Zugang zum Extensionsgedächtnis

\footnotetext{
${ }^{1}$ Die Dämpfung wird in der PSI-Theorie mit einer Klammer um das Plus oder das Minus ausgedrückt: $\mathrm{A}(+)$ bedeutet gedämpfter positiver Affekt; $\mathrm{A}(-)$ bedeutet herunterregulierter negativer Affekt.
} 
bzw. zum mit ihm verbundenen Selbstsystem; der so angebahnte Zugang zum Selbstsystem (Erfahrungsschatz, Bedürfnisse und Werte) unterstützt den konstruktiven Umgang mit negativen Empfindungen, indem bspw. durch eine sinnstiftende Bedeutungszuschreibung die Integration von ambivalenten Eindrücken erfolgen kann (Kuhl 2001, S. 174).

In den folgenden Abschnitten werden unter Einbezug der Notation der Affektregulation vier Leadershipstile von Schulführungskräften funktionsanalytisch (d.h. vor dem Hintergrund der Interaktion der psychologischen Makrosysteme; Kuhl 2001) abgeleitet und beschrieben.

\subsubsection{Visionär-begeisternder Stil: Kraft aus der Begeisterung und der erhofften positiven Außenwirkung}

Ein durch den positiven Affekt $(\mathrm{A}+)$ unter Beteiligung des Extensionsgedächtnisses /Selbstsystems [A(-)] charakterisierter Führungsstil besteht darin, dass Schulführungskräfte Entwicklungsprozesse von sich aus in Gang setzen und andere für ihre Ziele gewinnen wollen; die Energie dafür kommt hauptsächlich aus der Begeisterung für ein Ideal und/oder aus dem Bedürfnis, im Außen ein markantes, von anderen unübersehbares Signal setzen zu wollen, das Bewunderung und Anerkennung einbringt. Aufgrund ihres charismatischen (nicht-reflexiven) Agierens fallen ihnen Mitarbeiter/innen, die sich von dem von ihnen entfachten Schwung nicht ergreifen lassen, nicht negativ auf; solche Schulführungskräfte sind eher von der Zuversicht getragen, dass sich die Zurückhaltenden oder die Gegner/innen zu einem späteren Zeitpunkt anschließen würden (Annäherungsorientierung). Ein solcher "integrativer Motivationsstil" ("integrativ" deshalb, weil die Betreffenden in der optimistischen Überzeugung agieren, alle Erwartungen, Bedürfnisse und Wünsche aus dem Außen in ihrem Handeln zu erfüllen; vgl. Kuhl et al. 2010) zeichnet sich durch eine hohe Anfangsstabilität aus; d. h. dass solchermaßen motivierte Schulführungskräfte Entwicklungsvorhaben auch unter objektiv betrachtet ungünstigen Rahmenbedingungen in Gang setzen (denen sie aber keine Aufmerksamkeit schenken). Sollte der Widerstand aber wachsen und die Umsetzung des Vorhabens unübersehbar gefährden, gehen solche Schulführungskräfte "aus dem Feld" (geringe Endstabilität dieses Motivationsstils). Es wird dabei versäumt, dem Widerstand rechtzeitig durch Adaptionen des Entwicklungsvorhabens Rechnung zu tragen.

\subsection{2 (Latent) ängstlich-zielfixierter Stil: Sorge vor dem Scheitern des Vorhabens}

Im Falle eines durch den negativen Affekt (A-) charakterisierten Führungsstils fühlt sich die Schulführungskraft alternativlos in der Pflicht, bestimmte Ziele vorzugeben und die Mitarbeiter/innen - wenn nötig über Maßnahmen der Kontrolle, des Drucks oder der dezidierten Verpflichtung - dazu zu bringen, sich gewissenhaft und loyal am aufgesetzten Prozess zu beteiligen und ein Scheitern dieses Prozesses abwehren zu helfen (Vermeidungsorientierung). Die Entwicklungsziele, die im Fall eines solchen Leadershipstils verfolgt werden, bestehen darin, eine für den Schulstandort drohende Gefahr abzuwenden (z. B. Maßnahmen, um dem Rückgang der Anmeldezahlen entgegenzuwirken) oder eine von einer höheren Hierarchieebene angeordnete Aktivität (top-down) am Schulstandort gewissenhaft auszuführen. Charakteristisch für diesen Stil ist, dass solche Entwicklungsprozesse eher aktionistisch, d. h. wenig kontextsensibel (also ausgerichtet an der Frage: "Wovon wird am Schulstandort mehr bzw. weniger gebraucht?") durchgezogen werden; freilich ist bei einem solchen Stil die Gefahr gering, dass der Entwicklungsprozess - etwa bedingt durch ungünstige äußere Umstände (vgl. den visionär-begeisternden Stil) - ins Stocken gerät oder überhaupt versandet; die Schulführungskraft würde auf solche Tendenzen unmittelbar mit Appellen, sich anzustrengen und Durchhaltevermögen zu zeigen, reagieren (also hohe Endstabilität); aufgrund des hoch aktivierten negativen Affekts (A-) ist sie - im Unterschied zu Personen im visionär-begeisternden Stil - für solche Prozessphänomene ja außerordentlich sensibel.

\subsubsection{Passiv-vermeidender Stil: Ohnmachtsgefühle verhindern das Initiieren von Aktivitäten}

Hat eine Schulführungskraft die Zuversicht verloren, über Maßnahmen welcher Art auch immer den Zustand der Schule stabilisieren oder verbessern zu können, ist sie durch einen passiv-vermeidenden Führungsstil zu charakterisieren. Der Stress generierende negative Affekt (A-) verbindet sich im Fall dieses Führungsstils mit dem gedämpften positiven Affekt $[\mathrm{A}(+)]$, der eine Handlungshemmung bedeutet. Im Einzelnen sind auch bei dieser Affektkonfiguration Ressourcen für die Führung einer Schule und die Leitung eines Projektes zu finden: Die schon angesprochene ausgeprägte Fehlersensibilität und der Zugang, unangenehme Situationen auszuhalten und zu erdulden, schützen etwa auch vor einer überschnellen Übernahme von zentral verordneten Reformen, die im Bildungsbereich oft auch inflationär und in 
kurzen Abständen erfolgen (Clement 2014; Terhart 2013). Indirekt kann dieser Leadershipstil aber auch Entwicklungsprozesse unterstützen - etwa dann, wenn Initiativen aus dem Kollegium kommen und Lehrpersonen weitgehend autark in der Umsetzung und Verankerung an der Schule agieren, weil die Schulführungskraft aufgrund ihrer Disposition dabei nicht interveniert.

\subsubsection{Selbstgesteuerter Stil: am Ideal orientiert, konstruktiv mit Widerstand umgehend und den günstigen Augenblick (Kaıpóৎ—Kairòs) nutzend}

Im Unterschied zu MLQ (Bass und Avolio 1995a) ist PSItheoretisch ein so genannter selbstgesteuerter Leadershipstil abgrenzbar, der die Einseitigkeit des visionär-begeisternden oder des (latent) ängstlich-zielfixierten Leadershipstils ausbalanciert: Insbesondere für den Fall etwa, dass es im Kollegium einen Widerstand gegen Entwicklungsvorhaben gibt, ermöglicht dieser Leadershipstil einen konstruktiven Umgang damit, und zwar über folgendes Agieren: Bedenken werden nicht ignoriert (wie im Fall des visionär-begeisternden Leadershipstils) und auch nicht durch Zwang oder Verpflichtung beseitigt (wie im Fall des [latent] ängstlichzielfixierten Leadershipstils), sondern ernst genommen und als Ausgangspunkt für eine Adaptation des Entwicklungsziels und des -plans genutzt. Dieser Leadershipstil, der gerade für Leitungspersonen von pädagogischen Institutionen von Bedeutung ist (Hofmann 2020), weil Schulführungskräfte damit eine wichtige Modellfunktion für pädagogische Führung anbieten, findet sich bislang in Führungsstilinventaren nicht. Der selbstgesteuerte Leadershipstil ist dadurch gekennzeichnet, dass eine Schulführungskraft imstande ist, sich in einer bestimmten Berufssituation affektmäßig so zu disponieren, dass ihr eine authentische und kontextsensible Bewältigung der Situation gelingt; aufgrund der Situationsgebundenheit der Frage, wie selbstgesteuertes Agieren affektkonfiguriert ist, kann im Unterschied zu den anderen drei Stilen auf keine fixe Affektkonstellation verwiesen werden. Besonders kennzeichnend ist dabei aber die hoch ausgeprägte Fähigkeit zur emotionalen Dialektik: Der negative Affekt (etwa die Wahrnehmung von Widerständen, Ablehnung, Ängsten [im Kollegium]) kann gerade unter Stressbedingungen herabreguliert werden. Dieser psychische Prozess der Selbstberuhigung (Kuhl 2010, S. 41) ermöglicht dabei den Zugang zum Selbstsystem (mit dem Extensionsgedächtnis assoziiert; vgl. Kuhl 2010, S. 488), in dem eigene Bedürfnisse, Werte und Fähigkeiten repräsentiert sind. Dies ermöglicht der Schulleitung einen integrativkonstruktiven Umgang mit Widerständen gegen Entwicklungsvorhaben im Lehrer/innenkollegium. Kennzeichnend ist dabei auch eine besondere Aufmerksamkeit für partizipative Entscheidungsfindungen, die oft auch eine Integration von widersprüchlichen Meinungen und Ansichten erfordert
(Hofmann und Salzgeber 2017). Schulführungskräfte sind, vermittelt über diesen selbstgesteuerten Leadershipstil, fähig, über bestimmte Entwicklungsziele mit dem Kollegium einen Konsens herzustellen und eine für den Schulstandort passende und realisierbare Implementation in Gang zu setzen; eine solche Implementation integriert die beiden Pole Ideal und Realität. Ist also im Fall des visionärbegeisternden Leadershipstils die Gefahr gegeben, mit der Implementation eines hehren Ideals zu scheitern und im Fall des (latent) ängstlich-zielfixierten Leadershipstil die Wahrscheinlichkeit hoch, sich für großteils selbstfremde Ziele (z. B. von oben vorgegebene Ziele) instrumentalisieren $\mathrm{zu}$ lassen, gelingt es über den selbstgesteuerten Leadershipstil, ein Entwicklungsziel - möglicherweise auch in einer adaptierten Fassung - in den Schulalltag einzubringen und diesen Alltag damit funktionaler zur ganzheitlichen Entwicklung von Schüler/innen zu machen.

\section{Ziel der vorliegenden Studie}

In dieser Validierungsstudie soll das Instrumentarium $S E L F \_S F K$ nach der Darstellung der theoretischen Fundierung in Bezug auf dessen psychometrische Qualität überprüft werden. Durch die Entwicklung eines neuen Instrumentariums erhoffen sich die Autoren ein valides Instrument zur Verfügung zu stellen, das in bildungswissenschaftlichen Untersuchungen von Schulentwicklungsprozessen und in der Evaluation von Schulleitungshandeln eingesetzt werden kann. Die motivationspsychologische Fundierung und die Spezifizierung auf den Schulkontext sollen dabei die Mängel bisher etablierter Erhebungsinstrumente beheben und einen ersten Baustein für ein umfassenderes Screening darstellen.

Im empirischen Teil sollen folgende Hypothesen geprüft werden:

$\mathrm{H}_{1}$ : In Anlehnung an die theoretische Konzeption gehen wir von einer vierfaktoriellen Struktur aus, welche sich gegenüber aggregierten Lösungen psychometrisch bewährt.

$\mathrm{H}_{2}$ : Wir erwarten eine Messinvarianz in Bezug auf zentrale demographische Variablen wie das Geschlecht und die Berufserfahrung der Lehrpersonen, die Schulform und den Kontext der Schule (bspw. hinsichtlich eines attestierten Entwicklungsbedarfs), um das Instrumentarium in weiterführenden Studien für Unterschiedsmessungen in Bezug auf die Qualität des Schulleitungshandelns heranziehen zu können.

$\mathrm{H}_{3}$ : Das neu entwickelte Instrument weist korrelative Konvergenzen zu etablierten Leadershipskalen (MLQ; Bass und Avolio 1995a) sowie zu Selbsteinschätzungen in Drittvariablen auf, welche emotionale und motivationale Erlebensfaktoren in Bezug auf Schulent- 
wicklungsmaßnahmen messen (Eyal und Roth 2011). Dabei erwarten wir, dass der selbstgesteuerte Leadershipstil aufgrund der partizipativen Grundhaltung und der Berücksichtigung der Sichtweisen den höchsten Korrelationskoeffizienten zur Befriedigung des Bedürfnisses nach Autonomie aufweist, während der (latent) ängstlich-zielfixierte Stil in einem positiven Zusammenhang mit der Frustration des Bedürfnisses nach Autonomie steht (Gilbert und Kelloway 2014).

\section{Methode}

\subsection{Stichprobe}

Die Stichprobe umfasst $n=901$ Lehrpersonen an 75 Schulen der Primar- und Sekundarstufe, die im Rahmen von zwei Evaluationsstudien die eigene Schulführungskraft eingeschätzt haben. 52\% der Stichprobe umfasst Lehrpersonen an Schulen, welche auf Grundlage von Defiziten im nationalen Bildungsmonitoring an einer verpflichtenden Schulentwicklungsinitiative ("Grundkompetenzen absichern - GruKo"; vgl. Sobanski 2019) teilgenomen haben. 445 weitere Lehrpersonen (48\%) wurden über eine Weiterbildunginitiative für Schulführungskräfte zu einer Fragebogenerhebung eingeladen. Die Teilnahme an beiden Evaluationsstudien war freiwillig. Die Lehrpersonen wurden über ein Schreiben an die Schulleitungen erreicht und erhielten einen schulspezifischen Zugangstoken für die Erhebungsplattform. Die Zuordnung zu den Daten der Schulleitung bzw. die genestete Struktur der Daten wurde anhand der Identifikationsnummer des Schulstandortes vorgenommen und in den weiterführenden Analysen berücksichtigt.

Die Stichprobe setzte sich zu $40.3 \%$ aus Lehrenden der Primarstufe (Volksschule) und zu 59.7\% aus Lehrenden der Sekundarstufen I und II zusammen. Die Angaben in Bezug auf das Geschlecht ergaben eine Verteilung von $75.7 \%$ weiblichen und 24.3\% männlichen Proband/innen. Für die Angaben zur Berufserfahrung in Jahren erzielte die Variable eine Streuung von null bis 46 und einen Median von 14 Jahren.

\subsection{Instrumente}

Das hier zu validierende Erhebungsinstrument SELF_SFK operationalisiert vier grundlegende Stile von Leadershipverhalten in Bezug auf die Initiation und Leitung von Schulentwicklungsprozessen. Die theoretische Ausdifferenzierung der Stile stützt sich auf die Affektkonfigurationen der PSITheorie, welche jeweils eine Aktivierung [+, - ] und Hemmung $[(+),(-)]$ des positiven und des negativen Affektes postuliert (Kuhl 2001, 2010).
Zur Operationalisierung der Dimension visionär-begeisternder Leadershipstil wurde - teils auch in gedanklicher Anlehnung an die Stile der charismatischen Führung (Shamir et al. 1993) und des 'idealized behaviors' (Bass und Avolio 1995b) - insbesondere auf Formulierungen geachtet, die ein Handeln beschreiben, das durch positive Grundstimmung und positiver Affektgeladenheit Begeisterung erzeugen will. Dabei stehen primär die Schulführungskraft (SFK) und ihre Ideen und Vorstellungen im Mittelpunkt der Aufmerksamkeit und der inhaltlichen Ausrichtung des Prozesses; Widerstand oder Kritik werden nicht als solche wahrgenommen. Im (latent) ängstlich-zielfixierten Leadershipstil stehen ebenso primär die Ziele der SFK im Mittelpunkt; diese sind aber nicht kreativ generiert oder entsprechen deren Idealvorstellungen, sondern stammen allermeist aus Anordnungen übergeordneter Stellen oder erweisen sich für den Schulstandort als notwendig; um diese Ziele zu erreichen, setzt die Schulleitung auf kontrollierende und dominanzorientierte Handlungsweisen, mittels derer Fehler und als problematisch eingeschätzte Prozesse rasch korrigiert werden. Es geht der SFK darum, Ergebnisse - bisweilen auch gegen den Willen der Lehrpersonen - effektiv durchzusetzen. Bei Bedarf wird dabei auch auf die formelle oder institutionelle Hierarchie rekurriert, um die eigene Autorität für die Anordnung dieser Ziele zu bekunden. Demgegenüber bedeutet der passiv-vermeidende Leadershipstil, dass der SFK zwar auch problematische Entwicklungen, Fehler und Defizite an der Schule besonders präsent sind, sie aber keine Tatkraft aufbringen kann, um hier aktiv einzuschreiten. Dies ist auch daran gelegen, dass die Angst vor einer (weiteren) schmerzlichen Ohnmachtserfahrung größer ist als die Zuversicht, konstruktive Entwicklungen in Gang bringen zu können. Im selbstgesteuerten Leadershipstil werden jene Handlungsweisen abgebildet, die darauf hinweisen, dass eine Schulführungskraft im Stande ist, für die Schule wichtige Entwicklungsziele zu benennen, gleichzeitig dagegen auftretende Widerstände und negative Ereignisse wahrzunehmen und in einer solchen Konstellation eine konstruktive Lösung anzustreben. Wesentlich ist dabei auch der Aspekt der Partizipation des Lehrer/innenkollegiums, zum einen um den Schulentwicklungsprozess realitätsnahe zu gestalten und zum anderen, um auf authentische Art und Weise die integrative Haltung beim Umgang mit Konflikten und Widerständen umzusetzen.

Die insgesamt 16 Items wurden in randomisierter Reihenfolge auf einer Likert-Skala mit den Extremausprägungen $1=$ "stimmt nicht" bis $7=$ "stimmt genau" bewertet. Je ein Beispielitem und die Reliabilitätswerte der Skalen sind in Tabelle 4 abgebildet.

Zur Einschätzung der Konstruktvalidität wurde das neu entwickelte Instrument gemeinsam mit ausgewählten Skalen des Full-Range-Models (Bass und Avolio 1995a) getestet: 
Tabelle 3 Modellvergleich der konfirmatorischen Faktorenanalysen (CFA)

\begin{tabular}{lrrrrrrrr}
\hline Modell & \multicolumn{1}{c}{$\chi^{2}$} & $d f$ & $C F I$ & TLI & SRMR & RMSEA & AIC & BIC \\
\hline Vier-Faktoren-Lösung & 380.26 & 98 & 0.957 & 0.947 & 0.049 & 0.063 & 47,606 & 47,865 \\
Aggregierte zwei-Faktoren-Lösung\# & 582.46 & 101 & 0.928 & 0.914 & 0.053 & 0.073 & 47,839 & 48,083 \\
Agg. zwei-mal-zwei-Faktoren-Lösung & 792.65 & 103 & 0.898 & 0.881 & 0.059 & 0.095 & 48,082 & 48,318 \\
Aggregierte ein-Faktor-Lösung & 1103.31 & 104 & 0.847 & 0.824 & 0.071 & 0.103 & 48,502 & 48,733
\end{tabular}

$\chi^{2}$ robuster $\mathrm{Chi}^{2}$-Quadrat nach Satorra und Bentler (2001), $d f$ Freiheitsgrade, $C F I$ comparative fit index, TFI Tucker-Lewis index, SRMR standardized root mean square residual, RMSEA root mean square error of approximation, AIC Akaike Information Criteria, BIC Bayesian Information Criterion

\#Aggregierte Lösung der zwei Faktoren mit der höchsten Kovarianz
$\mathrm{Zu}$ diesem Zweck wurden fünf Dimensionen aus dem Multifactor Leadership Questionnaire (Full-Range-Model; Bass and Avolio 1995a, b) ausgewählt und mittels kleinerer sprachlicher Anpassungen (etwa "Lehrpersonen" anstelle von "Mitarbeitern") adjustiert. Im Einleitungssatz wurde der Fokus auf Schulentwicklungsaktivitäten spezifiziert und je nach regionalem Gebrauch wurden die Items mit dem Fragestamm "Mein/e Schulleiter/in" oder "Mein/e Schuldirektor/in" eingeführt. Die insgesamt 20 Aussageitems wurden auf einer siebenstufigen Likert-Skala ( $1=$ "trifft gar nicht zu"; 7 = "trifft voll und ganz zu") beantwortet. Für das transaktionale Spektrum wurden das Management by Exception Passive (MEP) und das Management by Exception Active (MEA) über je vier Items erhoben [Beispielitem: ... versäumt es, sich um die Probleme zu kümmern, bis sie wirklich ernst geworden sind. (MEP); ... verfolgt alle Fehler konsequent. (MEA)]. In der vorliegenden Stichprobe erreichte MEP eine gute interne Konsistenz $(\omega=0.84)$, das MEA akzeptable Reliabilitätswerte $(\omega=0.65)$. Für das Spektrum der transformationalen Leadershipstile wurden drei Dimensionen zu je vier Items beantwortet: Intellectual Stimulation (InS; Beispielitem: ... bringt Lehrpersonen dazu, Probleme aus verschiedenen Blickwinkeln zu betrachten.), Individual Consideration (InC; Beispielitem: ... erkennt individuelle Bedürfnisse, Fähigkeiten und Ziele von Lehrpersonen.) und Inspirational Motivation (InM; Beispielitem: ... formuliert eine überzeugende Zukunftsvision.). Alle Subskalen der transformationalen Leadership erzielten sehr zufriedenstellende Reliabilitätsmaße ( $\omega$ zwischen 0.90 und 0.94). Auf Grundlage einer konfirmatorischen Faktorenanalyse (CFA) wurde die Modellgüte für die verwendete Fünf-FaktorenLösung des Full-Range-Models geprüft und erzielte einen diskreten Fit: $\chi^{2}(160)=843.93 ; T L I=0.921 ; C F I=0.933$; RMSEA $=0.078 ;$ SRMR $=0.118$.

Zur Prüfung der Konstruktvalidität wurde die Befriedigung und Frustration des psychologischen Grundbedürfnisses nach Autonomie mit der validierten deutschsprachigen Version der German Basic Psychological Need Satisfaction and Frustration Scale (Heissel et al. 2018) erhoben und für den Kontext der Schulentwicklungsarbeit bei einzelnen Formulierungen spezifiziert. Beispielitems:
"... fühle ich mich gezwungen, viele Dinge zu tun, die ich mir selbst nicht aussuchen würde”; “... habe ich das Gefühl, dass meine Entscheidungen widerspiegeln, was ich wirklich will". Die Beantwortung erfolgt auf einer siebenstufigen Ratingskala mit dem Antwortformat 1 = "stimmt gar nicht" bis $7=$ "stimmt ganz genau". Beide Skalen erzielten gute bis sehr gute Werte für die Frustration (McDonalds $\omega=0.86$ ) und Befriedigung $(\omega=0.83)$.

\subsection{Analysen}

Für die deskriptiven Analysen wurden das Framework $R$ (R Core Team 2017) und die darauf basierende Anwendungssoftware jamovi (The jamovi project 2019) verwendet. Zur Berechnungen der Faktorenanalysen unter Einbezug der Mehrebenenstruktur sowie zur Schätzung der ICC wurden in den Analysen die Packages lavaan (Rosseel 2012) und multilevel (Blisse 2016) herangezogen. Fehlende Werte wurden durch die Funktion der full information maximum likelihood (FIML) berücksichtigt und in der Modellberechnung implementiert. Für eine verzerrungsfreie Schätzung der Parameter wurde die genestete Struktur der Lehrpersonen an $k=75$ durch eine Clusterungsvariable (dieselbe Schulleitung) und in den Analysen durch den maximum likelihood robust (MLR) Schätzer berücksichtigt (Musca et al. 2011). Auch für den Modellvergleich wurde der hierarchischen Datenstruktur durch den Einbezug des skalierten Chi-Quadrat-Tests nach Satorra und Bentler (2001) Rechnung getragen.

\section{Ergebnisse}

\subsection{Faktorenstruktur}

Als erster Validierungsschritt wurde mittels einer konfirmatorischen Faktorenanalyse (Brown 2006) die Struktur des Instrumentes an der Stichprobe von $n=901$ Lehrpersonen untersucht. Ausgehend vom theoretischen Modell werden vier Stile postuliert, welche sich durch eine VierFaktoren-Struktur abbilden lassen. Auf Grundlage eines 
Tabelle 4 Itemwortlaut und psychometrische Werte für die Stichprobe der Lehrpersonen $(n=901)$

\begin{tabular}{|c|c|c|c|c|c|c|}
\hline Beispielitem & $\alpha$ & $\omega$ & $M$ & $S D$ & $\lambda$ & $I C C$ \\
\hline Visionär-begeisternder Leadershipstil & 0.89 & 0.89 & 5.13 & 1.49 & & 0.26 \\
\hline ... präsentiert ihre/seine zukunftsgerichtete Vision für unsere Schule & & & 5.26 & 1.63 & 0.80 & 0.21 \\
\hline PA 2 & & & 5.15 & 1.87 & 0.84 & 0.25 \\
\hline PA 3 & & & 5.40 & 1.69 & 0.84 & 0.25 \\
\hline PA 4 & & & 4.95 & 1.64 & 0.79 & 0.17 \\
\hline (Latent) ängstlich-zielfixierter Leadershipstil & 0.69 & 0.70 & 3.51 & 1.34 & & 0.24 \\
\hline ... wehrt Lehrpersonen ab, wenn deren Vorschläge ihrem/seinem Plan nicht entsprechen & & & 3.27 & 1.96 & 0.81 & 0.25 \\
\hline NA 2 & & & 4.32 & 1.86 & 0.51 & 0.16 \\
\hline NA 3 & & & 3.15 & 1.75 & 0.61 & 0.13 \\
\hline NA 4 & & & 3.26 & 1.89 & 0.46 & 0.11 \\
\hline Passiv-vermeidender Leadershipstil & 0.83 & 0.83 & 2.32 & 1.27 & & 0.17 \\
\hline ... denkt über keine Entwicklungsziele nach, weil damit die Lage der Schule nicht besser wird & & & 2.06 & 1.49 & 0.86 & 0.09 \\
\hline VE 2 & & & 2.54 & 1.64 & 0.86 & 0.10 \\
\hline VE 3 & & & 2.21 & 1.55 & 0.83 & 0.16 \\
\hline VE 4 & & & 2.48 & 1.62 & 0.87 & 0.11 \\
\hline Selbstgesteuerter Leadershipstil & 0.90 & 0.90 & 5.00 & 1.50 & & 0.31 \\
\hline $\begin{array}{l}\text {... greift Entwicklungsinitiativen des Lehrerkollegiums auf und generiert gemeinsam mit den } \\
\text { Lehrpersonen Schulentwicklungsziele für die Schule }\end{array}$ & & & 5.18 & 1.66 & 0.70 & 0.24 \\
\hline SE 2 & & & 4.70 & 1.78 & 0.70 & 0.20 \\
\hline SE 3 & & & 4.80 & 1.79 & 0.82 & 0.30 \\
\hline SE 4 & & & 5.30 & 1.63 & 0.71 & 0.25 \\
\hline
\end{tabular}

$\alpha$ Cronbachs Alpha, $\omega$ McDonalds Omega, $M$ Item- bzw. Skalenmittelwert, SD Standardabweichung, $\lambda$ standardisierte Faktorenladung, ICC Intraklassenkoeffizient

Modellvergleiches weist die theoretisch postulierte Struktur den besten Fit auf (vgl. Tabelle 3): $\chi^{2}=380.26 ; d f=98$; $C F I=0.957 ; T L I=0.947 ; S R M R=0.049 ; R M S E A=0.057$. Der Modelltest war signifikant $(p<0.01)$, was jedoch auf Grundlage der großen Stichprobe als nicht problematisch einzuschätzen ist (Bergh 2015).

Komplementär wurden auch drei weitere aggregierte Faktorenmodelle erstellt und einem Modellvergleich unterzogen. Trotz teils akzeptabler Prüfwerte (vgl. Tabelle 3) für eine aggregierte Lösung aus den beiden vom positiven Affekt geprägten Stilen weist ein Modellvergleich der Chi-QuadratStatistik auf eine signifikant bessere Lösung $(p<0.001)$ der postulierten vier-Faktoren-Struktur gegenüber den anderen Lösungen hin, was auch an den Vergleichsindizes AIC und BIC (Kuha 2004) abgelesen werden kann.

Alle Items der Subskalen (vgl. Tabelle 4) wiesen signifikante Faktorenladungen auf. Bis auf ein Item, das aufgrund der Negation rekodiert wurde und eine geringe Ladung von 0.45 erreichte, erzielten die standardisierten Koeffizienten aller anderen Items einen Ladungsparameter zwischen 0.81 und 0.93. Die Subskalen weisen eine akzeptable bis hohe interne Konsistenz auf, was an den Werten der Cronbachs Alphas zwischen 0.78 und 0.94 abgelesen werden kann. Auch die Koeffizienten für das McDonalds Omega, welche die latente Faktorenladung zur Schätzung der Reliabilität berücksichtigen (Revelle und Zinbarg 2009), erzielten hohe Werte zwischen 0.82 und 0.94 .

Auf latenter Ebene finden sich signifikante $(p<0.001)$ Kovarianzen zwischen den Subdimensionen des Inventars, welche in Abbildung 2 vorzufinden sind. Zwischen dem Leadershipstil visionär-begeisternd wurde eine hohe Kovarianz zum selbstgesteuerten Stil erzielt $(r=0.90)$, während negative Kovarianzen zum (latent) ängstlich-zielfixierten $(r=-0.59)$ und zum passiv-vermeidenden Stil $(r=-0.73)$ vorliegen. Der passiv-vermeidende Leadershipstil weist eine moderate bis hohe Kovarianz mit dem (latent) ängstlichzielfixierten Leadershipstil auf $(r=0.62)$; beide vom negativen Affekt geprägten Stile stehen wiederum in einem negativen Zusammenhang zum selbstgesteuerten Leadershipstil $(r=$ zwischen -0.70 und -0.75$)$.

\subsection{Messinvarianz}

Die Einschätzungen zum Leadershiphandeln wurden hinsichtlich der Messinvarianz für die Variablen Geschlecht und Schulform der teilnehmenden Lehrpersonen geprüft. Die Berechnung der Messinvarianz erfolgt dabei stufenweise (vgl. Wang et al. 2018): konfigural, ohne Einschränkung der Koeffizienten; metrisch, durch Gleichsetzung der 


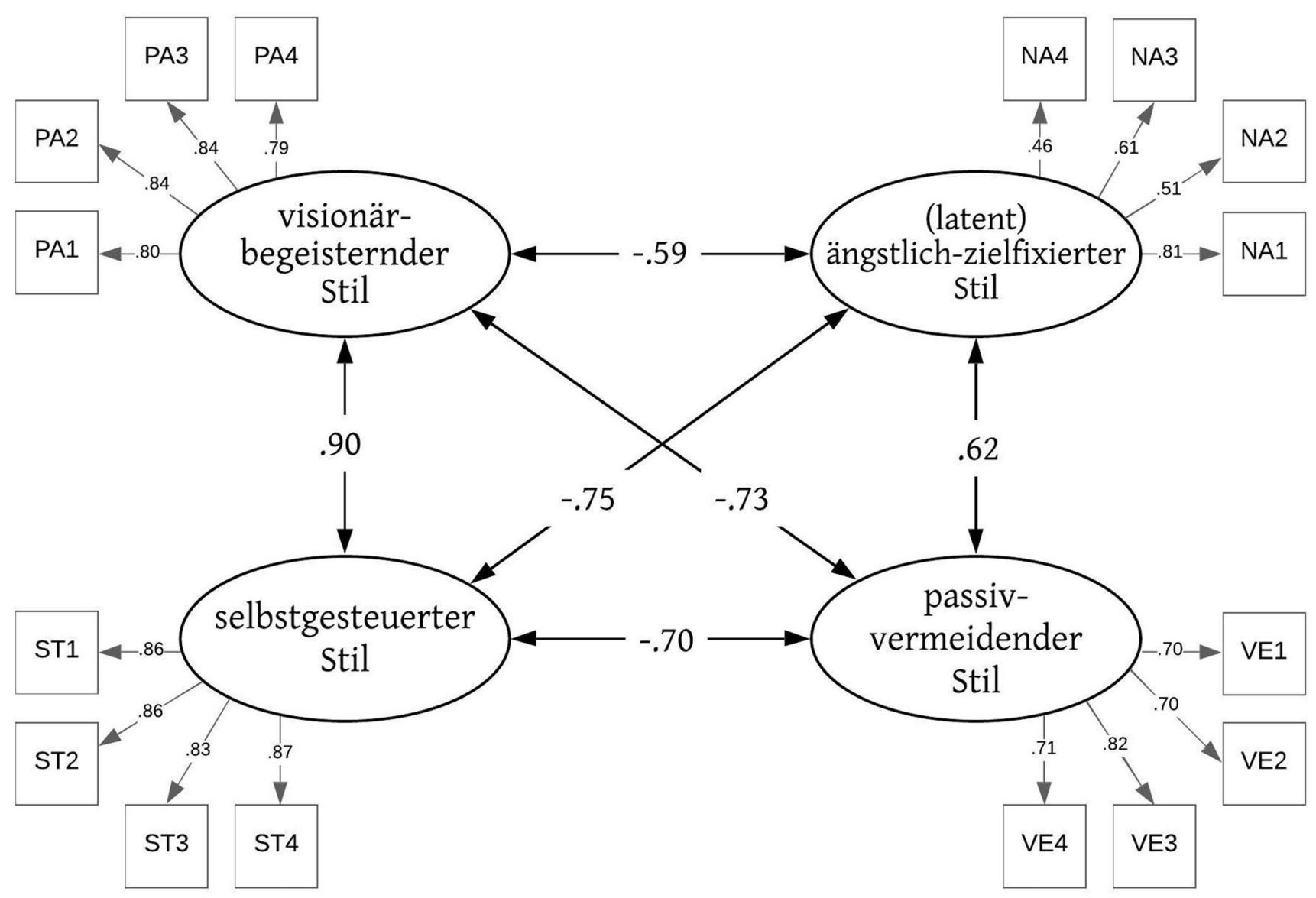

Abbildung 2 Latente Faktorenstruktur des Leadershipinventar-Moduls

Tabelle 5 Messinvarianz für Geschlechterunterschiede

\begin{tabular}{lllllll}
\hline Geschlecht & $\chi^{2}$ & $d f$ & CFI & TLI & SRMR & RMSEA \\
\hline Konfigural & 380.26 & 98 & 0.957 & 0.947 & 0.049 & 0.063 \\
Metrisch & 514.57 & 208 & 0.955 & 0.948 & 0.057 & 0.063 \\
Skalar & 521.76 & 220 & 0.956 & 0.952 & 0.057 & 0.061 \\
\hline
\end{tabular}

Anmerkungen: siehe Tabelle 3

Faktorenladungen; und skalar, durch die Fixierung der Intercepts der Items.

Geschlecht: Die Prüfung des Basismodells, bei dem alle Parameter beider Geschlechtsausprägungen der Proband/ innen frei geschätzt wurden, erzielte einen sehr guten Modellfit. Weder die Fixierung der Faktorenladungen noch der Intercepts (vgl. Tabelle 5) führte zu einer signifikanten Verschlechterung des Modellfits $(p>0.05)$, weshalb von einer Messinvarianz in Bezug auf das Geschlecht der Teilnehmenden ausgegangen werden kann.

Schulform: Die Einschätzung der Leadershipstile wurde sowohl an Primar- wie an Sekundarschulen erhoben, die sich in einigen Facetten der Organisations- und Professionskultur (bspw. Klassenlehrpersonen vs. fachbezogene
Lehrkräfte, Größe des Lehrerkollegiums etc.) unterscheiden. Die Fixierung der Faktorenladungen (vgl. Tabelle 6) führte auf Grundlage des skalierten Chi-Quadrat-Tests nach Satorra und Bentler (2001) zu einem signifikanten Unterschied $\left(\Delta \chi^{2}=166.94 ; p<0.001\right)$ zum konfiguralen Modell. Der Unterschied bei den Vergleichsindizes $(\triangle C F I=0.009$; $\triangle S R M R=0.003$ ) ist jedoch sehr gering und kann auf Grundlage der Empfehlungen von Chen (2007, S. 501) als nicht bedeutsam interpretiert werden.

Schulkontext: $\mathrm{Zu} 52 \%$ setzte sich die Stichprobe aus Lehrpersonen zusammen, die derzeit an einer Entwicklungsinitiative für Schulen mit Defiziten in Lernstanderhebungen beschäftigt sind. Diesbezüglich wurde in einem dritten Schritt geprüft, ob in einem Vergleich zwischen 
Tabelle 6 Messinvarianz in Bezug auf die Schulform

\begin{tabular}{lllllll}
\hline Schulform & $\chi^{2}$ & $d f$ & $C F I$ & $T L I$ & SRMR & RMSEA \\
\hline Konfigural & 380.26 & 98 & 0.957 & 0.947 & 0.049 & 0.063 \\
Metrisch & 570.67 & 208 & 0.954 & 0.947 & 0.056 & 0.066 \\
Skalar & 576.98 & 220 & 0.948 & 0.943 & 0.057 & 0.066 \\
\hline
\end{tabular}

Anmerkungen: siehe Tabelle 3

\begin{tabular}{llrllll}
\hline Geschlecht & $\chi^{2}$ & $d f$ & $C F I$ & $T L I$ & SRMR & RMSEA \\
\hline Schulkontext & 380.26 & 98 & 0.957 & 0.947 & 0.049 & 0.063 \\
Metrisch & 521.48 & 208 & 0.953 & 0.946 & 0.056 & 0.064 \\
Skalar & 559.19 & 220 & 0.948 & 0.944 & 0.057 & 0.066 \\
\hline
\end{tabular}

Anmerkungen: siehe Tabelle 3
Tabelle 7 Messinvarianz in Bezug auf den Schulkontext
Lehrpersonen an Schulen mit und an Schulen ohne einen extern zugeschriebenen Entwicklungsbedarf Messinvarianz festgestellt werden kann. Der Chi-Quadrat Test erwies sich für beide untersuchten Konfigurationen, sowohl die Prüfung der metrischen $\left(\Delta \chi^{2}=136.93 ; p<0.05\right)$ wie auch der skalaren Invarianz $\left(\Delta \chi^{2}=180.22 ; p<0.001\right)$, als signifikant (Tabelle 7). Die Unterschiede bezüglich der Vergleichsindizes sind geringer als die cut-off Kriterien von Chen (2007, S. 501) und können damit als nicht bedeutsam interpretiert werden $(\triangle C F I<0.01 ; \triangle R M S E A<0.03$; $\triangle S R M R<0.015)$.

\subsection{Konstruktvalidität}

Zur Prüfung der Konstruktvalidität wurden die Dimensionen des neu entwickelten Inventarmoduls mit fünf ausgewählten Dimensionen des MLQ korreliert und mit der Bedürfnisbefriedigung und Frustration der Teilnehmer/innen in Bezug zur Teilnahme an Schulentwicklungsprojekten gesetzt (vgl. Tabelle 8).

Korrelationen der Subdimensionen des Leadershipinventars: Wie bereits in der latenten Faktorenstruktur ersichtlich, weist der Leadershipstil visionär-begeisternd auch auf manifester Ebene eine hohe Korrelation zum selbstgesteuerten Leadershipstil auf ( $r=0.80, p<0.001)$, während negative Korrelationskoeffizienten zum (latent) ängstlich-zielfixierten ( $r=-0.48, p<0.001)$, und passiv-vermeidenden Leadershipstil $(r=-0.63, p<0.001)$ vorliegen. Der passivvermeidende Leadershipstil weist eine moderate bis hohe Kovarianz mit dem (latent) ängstlich-zielfixierten Leadershipstil auf $(r=0.49, p<0.001)$; beide vom negativen Affekt geprägten Stile stehen wiederum in einem negativen Zusammenhang zum selbstgesteuerten Leadershipstil ( $r=$ zwischen -0.59 und $-0.60, p<0.001$ ) (Tabelle 8).

Konvergente Validität mit dem Full-Range-Leadershipinventar: Für den visionär-begeisternden Leadershipstil wird ein hoher und positiver Zusammenhang zu allen drei Subdimensionen der transformationalen Stile (Intellectual Stimulation, Inspirational Motivation und Individual Consideration; alle $r=0.81, p<0.001$ ) beobachtet. Zum Management by Exception Passive liegt ein moderater bis hoher, negativer Zusammenhang vor $(r=-0.56, p<0.001)$, während das Management by Exception Active keinen Zusammenhang zum visionär-begeisternden Leadershipstil aufweist. Der (latent) ängstlich-zielfixierte Leadershipstil hingegen weist zu allen Stilen korrelative Zusammenhänge auf: Er korreliert moderat bis hoch mit den Stilen der transaktionalen Leadership ( $r=0.57$ ME-Passiv; $r=0.34$, ME-Active; beide $p<0.001$ ), während für die transformationalen Stile negative Korrelationen zwischen $r=-0.56$ und $r=-0.46$ (alle $p<0.001$ ) berichtet werden. Analog dazu erzielt der passiv-vermeidende Leadershipstil die höchste Korrelation zum Management by Exception Passive ( $r=0.66, p<0.001$ ), einen kleinen, aber signifikanten Korrelationskoeffizienten zum Management by Exception Active $(r=0.11, p<0.01)$, während die transformationalen Stile in moderat negativen Zusammenhängen zwischen $\mathrm{r}=-0.57$ und $r=-0.53$ (alle $p<0.001)$ stehen. Demgegenüber weist der selbstgesteuerte Leadershipstil hoch ausgeprägte und positive Zusammenhänge zu den transformationalen Führungsstilen auf ( $r=$ zwischen 0.76 und $0.82, p<0.001$ ), wenn auch mit einem etwas geringeren Koeffizienten für die Inspirational Motivation im Vergleich zur Korrelation beim visionärbegeisternden Leadershipstil.

Korrelationen mit Drittvariablen: Die Ausprägungen des neu entwickelten Instrumentariums konnten mit dem motivationalen Erleben der Lehrpersonen in Zusammenhang gebracht werden. Dabei weist der selbstgesteuerte Leadershipstil die günstigsten Zusammenhänge auf und korreliert positiv mit der Befriedung ( $r=0.35$ ) und negativ mit der Frustration $(r=-0.36, p<0.001)$ des Bedürfnisses nach Autonomie; für den visionär-begeisternden Leadershipstil 
werden analoge Zusammenhänge berichtet $(r=0.28 \mathrm{bzw}$. $-0.22, p<0.001$ ), wenn auch von einem etwas geringeren Ausmaß des Koeffizienten. Demgegenüber stehen die vom negativen Affekt geprägten Stile in einem ungünstigen Zusammenhang zur BPNSF mit moderat negativen Zusammenhängen zur Bedürfnisbefriedigung ( $r=$ zwischen -0.23 und -0.21$)$ und einem positiven Zusammenhang zur Frustration der Autonomie ( $r=$ zwischen 0.27 und 0.39, $p<0.001$ ). Die Zusammenhänge der neu entwickelten Leadershipskalen zu den motivationalen Variablen sind etwas stärker ausgeprägt als die Korrelationen des Full-RangeInventars, bei dem die höchste Korrelation zur Bedürfnisbefriedigung von der Individual Consideration ( $r=0.32$, $p<0.001)$ bzw. vom Management by Exception Passive $(r=0.27, p<0.001)$ hin zur Bedürfnisfrustration erzielt wird (Tabelle 8).

\section{Diskussion und Ausblick}

In diesem Beitrag wurde die Entwicklung und Validierung eines Instrumentenmoduls zum Schulleitungshandeln im Kontext von Schulentwicklungsprozessen vorgestellt und für eine umfangreiche Stichprobe von Lehrpersonen validiert. Gegenüber schon vorhandenen Instrumentarien bietet SELF_SFK die Möglichkeit, das Schulleitungshandeln zugleich domänenspezifisch (Domäne: Entwicklung auf Organisationsebene zu stimulieren) und differenziert in Bezug auf den Leadershipstil abzubilden. Aufgrund einer konfirmatorischen Faktorenstrukturanalyse konnte die Dimensionierung durch vier Leadershipstile bestätigt werden. Dies ermöglicht, bei Schulleitungen vier Leadershipstile zu identifizieren: den (1) visionär-begeisternden, den (2) (latent) ängstlich-zielfixierten, den (3) passiv-vermeidenden und den (4) selbstgesteuerten Leadershipstil. Das Instrument erwies sich invariant in Bezug auf das Geschlecht der teilnehmenden Lehrpersonen. In Bezug auf die Schulstufe (Primar- vs. Sekundarstufe) und in Bezug auf den Schulkontext (mit bzw. ohne attestiertem/n Entwicklungsbedarf) wurden signifikante Unterschiede bezüglich des $\chi^{2}$-Modellfits berichtet; die Unterschiede bezüglich der Vergleichsindizes (CFI, TFI, SRMR und RMSEA) waren nur marginal und können in Anlehnung an die Kriterien von Chen (2007, S. 501) als nicht bedeutsam interpretiert werden.

In Bezug auf die Konstruktvalidität können durch Korrelationsberechnungen mit schon validierten Inventaren wie auch mit motivationalen Variablen inhaltliche Konvergenzen berichtet werden: Sowohl der visionärbegeisternde als auch der selbstgesteuerte Leadershipstil korrelieren mit den Substilen der transformationalen Leadership nach Bass und Avolio (1995a): Der selbstgesteuerte Leadershipstil wies dabei eine geringere Kovarianz

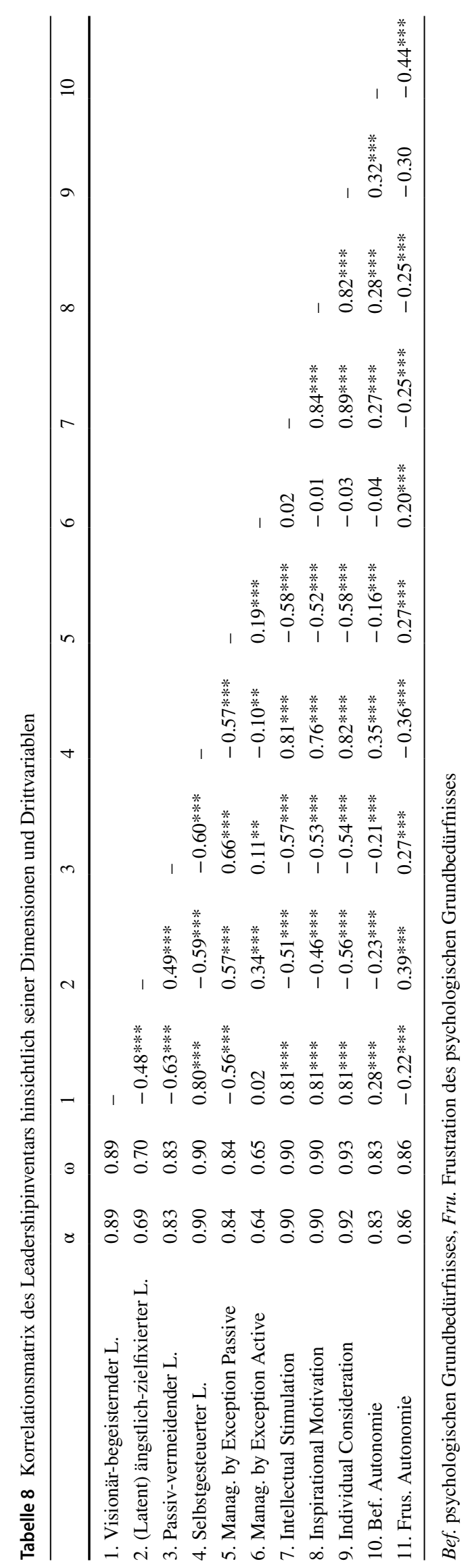


zur inspirational motivation auf, wodurch eine stärkere Ausbalancierung des positiven Affektes zum Ausdruck kommt. Darüber hinaus wies der selbstgesteuerte Leadershipstil die höchsten Zusammenhänge zum Bedürfnis nach Autonomie auf: Schulleitungen, welche einen selbstgesteuerten Zugang in der Initiierung von Schulprojekten wählen, räumen Lehrpersonen stärker die Möglichkeit ein, auch eigene Wünsche, Ziele und Zugangsweisen zu äußern. Entsprechend grenzt sich dieser Stil von den eher charismatischen Anteilen der transformationalen Führung ab, die schon zuvor als ein kritischer Bestandteil des FullRange-Models diskutiert wurden (Furtner und Baldegger 2013, S. 153; Yukl 1999). Bei charismatischen Führungskräften kann sich der Fokus von den allgemeinen Zielen für die Entwicklung der gesamten Organisation hin zu den Selbstinteressen und Machtbedürfnissen der Leitung verschieben. Mitarbeiter/innen werden durch eine hohe persönliche Identifikation mit der Führungskraft verleitet, eigene Bedenken oder Kritik am Vorhaben nicht zu äußern und selbstfremde Ziele als eigene zu übernehmen.

Eine solche Unterscheidung ist insofern von Bedeutung, (1) als es sich bei Schulentwicklungsprozessen um mittelbis langfristig angelegte Prozesse handelt, die sich oft über ein ganzes Unterrichtsjahr (und bisweilen darüber hinaus) erstrecken. Das bedeutet, dass die Frage, in welchem Ausmaß sich einzelne Lehrpersonen mit den Projektzielen identifizieren können, für die Motivation, sich an den Prozessen konstruktiv zu beteiligen, nicht unerheblich ist (Kwakman 2003). (2) Außerdem ist in diesem Zusammenhang zu bedenken, dass das Mitwirken von Lehrpersonen an Schulentwicklungsprozessen neben ihrer regulären Unterrichts- und der damit zusammenhängenden Organisationsarbeit bewältigt werden muss; dieser Umstand ist darüber hinaus im Kontext des Faktums zu sehen, dass Lehrpersonen die Schulentwicklungsarbeit im Vergleich zur Unterrichtstätigkeit noch nicht im selben Ausmaß und mit derselben Selbstverständlichkeit als regulären Arbeitsbereich ansehen, was ebenso motivationale Probleme mit sich bringen kann - insbesondere dann, wenn der Identifikationsgrad mit den Projektzielen aufgrund eines charismatischen Vorgehens der Schulführungskraft eher gering ist.

Die selbstgesteuerte Umsetzung von Schulentwicklungsvorhaben ist aus folgenden Gründen für die Schulkultur an einem Standort als relevant zu betrachten: Da Schulen in jüngerer Zeit aufgrund des Mangels an Schüler/ innen unter dieser Perspektive betrachtet zueinander in Konkurrenz stehen, ist die Gefahr gegeben, dass sie sich unter Orientierung an einem marktfähigen Merkmal profilieren wollen (Orientierung an Außenkriterien, die allerdings dem Zeitgeist und den Gesetzen des Marketings unterworfen sind). Für die Qualität der Schulkultur würde das bedeuten, dass bestimmte Entwicklungsziele nicht im
Dienst der Qualität der internen Prozesse (z. B. Interaktionsqualität zwischen Lehrenden und Lernenden) stehen, sondern im Dienst einer möglichst positiven Außenwahrnehmung. Damit würde eine authentische Umsetzung solcher Projektziele, die die Qualität der Schulkultur steigern, gefährdet sein, zumal der Fokus nicht darauf gerichtet ist, was im Blick auf die Schüler/innen pädagogisch geboten ist, sondern darauf, was in der öffentlichen Wahrnehmung Vorteile bringen kann.

\subsection{Limitationen}

Die hohe Kovarianz zwischen den latenten Konstrukten, die vom positiven Affekt geprägt sind, stellt einen Punkt dar, der bei der Komplettierung des Instrumentariums berücksichtigt werden soll: Bei der Operationalisierung der weiteren fünf Module der in Abschnitt 3.1 beschriebenen Systematik soll noch stärker auf die Unterscheidung zwischen einer unregulierten Umsetzung des positiven Affektes im visionär-begeisterten Leadershipstil und einer balancierten Umsetzung im selbstgesteuerten Leadershipstil eingegangen werden. Auch wenn eine hohe Kovarianz aus funktionsanalytischer Perspektive keine Redundanz der Konstrukte bedeutet (Kuhl 2001, S. 24), kann die gemeinsame Verwendung aller Dimensionen als Prädiktoren zu unerwünschten Supressionseffekten führen (Tabachnick und Fidell 2013, S. 122), welche die Bestimmung des Regressionskoeffizienten erschwert. Eine separierte Modellbildung mit je zwei kontrastierenden Stilen ermöglicht durch einen Modellvergleich mittels der Bestimmung der aufgeklärten Varianz, den Regressionseffekt der einzelnen Skalen zu eruieren.

Als weitere methodische Einschränkung sei angemerkt, dass die Anzahl der Lehrpersonen, die eine Einschätzung ihrer Schulleitung vorgenommen haben, innerhalb der jeweiligen Schulstandorte aufgrund unterschiedlicher Rücklaufquoten variiert hat. Neben der Berechnung des Übereinstimmungskoeffizienten (vgl. ICC Tabelle 4) soll daher in zukünftigen Analysen auch untersucht werden, ob eine niedrige Rücklaufquote eine Verzerrung der Einschätzung des Leadershiphandelns verursachen kann. Die Ergebnisse dieser Analyse können dann herangezogen werden, um den Einsatz des validierten Instrumentes für das Assessment des Leadershiphandelns einzelner Schulführungskräfte zu optimieren.

Des Weiteren sei abschließend auf eine metatheoretische Überlegung verwiesen, die insbesondere dann von Relevanz ist, wenn das entwickelte Inventar für Trainingszwecke grundlegend wird. Die Ableitung der Leadershipstile unter funktionsanalytischer Perspektive bedeutet eine Fokussierung auf psychologische Mikroprozesse, deren Erwerb einer trainingsmäßigen Spezifikation - im Sinne einer technologischen Theorie - bedarf (Astleitner 
2020). Das bedeutet, dass zusätzlich zur Konzeption eines solchen Inventars zur Entwicklungsstimulierung des selbstgesteuerten Leitungsstils oder eines kontextsensiblen Einsatzes der vier beschriebenen Stile der Entwurf eines pädagogischen Ansatzes notwendig ist, der auf die Förderung und Entwicklung von Selbstkompetenzen durch die Verbindung von persönlichkeitsdiagnostischen Instrumenten, reflexiven Übungen und Praxisbegleitung (vgl. Hofmann 2020) abzielt, wie auch in den Schlussfolgerungen näher erläutert wird.

\section{Schlussperspektive}

Durch die solide funktionsanalytische Ableitung und die ressourcenorientierte Perspektive der PSI-Theorie (Kuhl et al. 2010) bietet dieses Instrument ein breiteres Spektrum an Handlungsoptionen, die in der Weiterbildung und im Coaching von Schulführungskräften aufgegriffen werden können, um einen kontextsensiblen und wirksamen Umgang mit den Herausforderungen am Schulstandort zu unterstützen:

- So ermöglicht die Trennung der Aufgaben- von der Umsetzungsebene, die aufgrund der PSI-theoretischen Fundierung möglich wird, eine differenziertere Diagnostik (insbesondere dann, wenn auch die Skalen der restlichen Module vorliegen): Es können nicht nur Angaben über die Art der Umsetzung einzelner Aufgaben (im Falle dieses Moduls: der Schulentwicklungsaufgaben) gemacht werden, sondern es kann auch eruiert werden, wie es um die transsituationale Konsistenz des Leadershipstils bestellt ist; es könnte ja sein, dass einer Schulführungskraft die Umsetzung einer bestimmten Aufgabe im selbstgesteuerten Stil besser gelingt als die Umsetzung einer anderen Aufgabe. Ein solcher diagnostischer Befund wäre für die Konzeption von Trainingsmaßnahmen für Schulführungskräfte eine wertvolle Grundlage, um deren Entwicklung im Leitungshandeln andere Aufgaben betreffend zu unterstützen.

- Des Weiteren kann auf der Basis solcher individualdiagnostischer Ergebnisse in Coachings oder in Fortbildungssituationen darüber gesprochen werden, für welche Alltagssituationen etwa der visionär-begeisternde Stil durchaus viabel ist bzw. in welchen (freilich wohl bedeutend selteneren) Situationen selbst der (latent) ängstlich-zielfixierte Stil funktional ist (etwa wenn Gefahr in Verzug ist). Nicht immer gilt ja, dass der selbstgesteuerte Stil, der in Trainingssituationen freilich als Zielperspektive fungiert, weil ihn viele Schulführungskräfte zu wenig ausgebildet haben, zur konstruktiven Bewältigung einer Alltagssituation am besten passt; die Frage, welcher Stil vergleich- sweise viabler ist, kann ja nur aus der Kenntnis der Anforderungen der zu bewältigenden Situation und ihrer Rahmenbedingungen eruiert werden. Auch diese Unterscheidung wäre ein wichtiges Ziel in Fortbildungssituationen für Schulführungskräfte.

- Nicht zuletzt könnten, wenn alle Skalen zu allen Modulen für ein umfassendes Screening eingesetzt werden, aus intraindividuellen Differenzen des Stils einzelne Schulleitungsaufgaben betreffend Thesen zur entwicklungsrelevanten Frage aufgestellt werden, welche Aufgabenfelder bei einer Schulführungskraft mehr Begeisterung als Stress auslösen und wie es der Schulführungskraft gelingen kann, bei aller Begeisterung die Perspektive nicht aus den Augen zu verlieren, dass auch das Commitment des Kollegiums wichtig ist und bei allem Stress nicht in einen Tunnelblick zu rutschen und Schul- und Unterrichtsentwicklung aktionistisch zu betreiben, weil damit die Qualität der Prozesse erheblich beeinträchtigt würde. Beide genannten Risken können besser im (ganzheitlichen) Blick behalten werden, wenn Schulführungskräfte über die grundsätzliche Fähigkeit, im selbstgesteuerten Stil zu agieren, verfügen, was insbesondere unter Stressbedingungen auch für "Geübte" eine Herausforderung bleibt.

\section{Declarations}

Conflict of interest The authors declare that they have no conflict of interest to disclose.

Open Access Dieser Artikel wird unter der Creative Commons Namensnennung 4.0 International Lizenz veröffentlicht, welche die Nutzung, Vervielfältigung, Bearbeitung, Verbreitung und Wiedergabe in jeglichem Medium und Format erlaubt, sofern Sie den/die ursprünglichen Autor(en) und die Quelle ordnungsgemäß nennen, einen Link zur Creative Commons Lizenz beifügen und angeben, ob Änderungen vorgenommen wurden. Die in diesem Artikel enthaltenen Bilder und sonstiges Drittmaterial unterliegen ebenfalls der genannten Creative Commons Lizenz, sofern sich aus der Abbildungslegende nichts anderes ergibt. Sofern das betreffende Material nicht unter der genannten Creative Commons Lizenz steht und die betreffende Handlung nicht nach gesetzlichen Vorschriften erlaubt ist, ist für die oben aufgeführten Weiterverwendungen des Materials die Einwilligung des jeweiligen Rechteinhabers einzuholen. Weitere Details zur Lizenz entnehmen Sie bitte der Lizenzinformation auf http://creativecommons. org/licenses/by/4.0/deed.de.

\section{Literaturverzeichnis}

Altrichter H, Maag Merki K (eds) (2016) Handbuch Neue Steuerung im Schulsystem. Springer Fachmedien, Wiesbaden 
Astleitner H (2020) Alternative theoretical frameworks for educational interventions. In: Astleitner $\mathrm{H}$ (ed) Intervention research in educational practice. Waxmann, Münster, pp 19-37

Avolio BJ, Bass BM (2002) Developing potential across a full range of leadership. Cases on transactional and transformational leadership. Lawrence Erlbaum Associates, Mahwah

Bass BM, Avolio BJ (1994) Improving organizational effectiveness through transformational leadership. Sage, Thousand Oaks

Bass BM, Avolio BJ (1995a) MLQ multifactor leadership questionnaire technical report. Binghamton University, NY: Center for Leadership Studies

Bass BM, Avolio BJ (1995b) Theory of transformational leadership redux. Leadersh Q 6(4):463-478

Bergh D (2015) Chi-Squared test of fit and sample size. A comparison between a random sample approach and a Chi-Square value adjustment method. J Appl Meas 16(2):204-217

Blisse P (2016) Package 'multilevel'. https://cran.r-project.org/web/ packages/multilevel/index.html

Bogler R (2001) The influence of leadership style on teacher job satisfaction. Educ Adm Q 37(5):662-683. https://doi.org/10.1177/ 00131610121969460

Bonsen M (2016) Schulleitung und Führung in der Schule. In: Altrichter H, Maag Merki K (eds) Handbuch Neue Steuerung im Schulsystem. Springer Fachmedien, Wiesbaden, pp 301-323

Brauckmann S, Böse S (2018) Datengestütztes Schulleitungshandeln zwischen Anspruch und Wirklichkeit - Einsichten und Aussichten. In: Krainer K, Posch P, Rauch F (eds) Das Lernen von Lehrerinnen und Lehrern, Organisationen und Systemen. Festschrift zum 60. Geburtstag von Konrad Krainer. Waxmann, Münster, pp $165-178$

Brauckmann S, Eder F (2019) Führungsforschung im Bildungsbereich: Schulleitung im Spannungsfeld erweiterter Rechte und Pflichten. Z Bild 9(1):5-15. https://doi.org/10.1007/s35834-019-00242-6

Brauckmann S, Pashiardis P (2011) A validation study of the leadership styles of a holistic leadership theoretical framework. Int J Educ Manag 25(1):11-32. https://doi.org/10.1108/09513541111100099

Brown TA (2006) Confirmatory factor analysis for applied research (methodology in the social sciences). Guilford Press, New York

Burns JM (1978) Leadership. Harper \& Row, New York

Cambridge Dictionary (2014) Style. In: Cambridge Dictionary, Cambridge University Press. https://dictionary.cambridge.org/de/worte rbuch/englisch-deutsch/style

Chen FF (2007) Sensitivity of goodness of fit indexes to lack of measurement invariance. Struct Equ Model 14(3):464-504. https://doi. org/10.1080/10705510701301834

Clement J (2014) Managing mandated educational change. Sch Leadersh Manag 34(1):39-51. https://doi.org/10.1080/13632434.2013. 813460

Demski D (2017) Evidenzbasierte Schulentwicklung. Empirische Analyse eines Steuerungsparadigmas (Schulentwicklungsforschung). Springer, Wiesbaden

Eyal O, Roth G (2011) Principals' leadership and teachers' motivation. J Educ Adm 49(3):256-275. https://doi.org/10.1108/09578 231111129055

Furtner M, Baldegger U (2013) Self-Leadership und Führung. Theorien, Modelle und praktische Umsetzung. Springer Gabler, Wiesbaden

Gilbert SL, Kelloway EK (2014) Leadership. In: Gagné M (ed) The Oxford handbook of work engagement, motivation, and selfdetermination theory. Oxford Univ. Press, Oxford, pp 181-198

Göhlich M (2018) Geschichte der Organisationspädagogik. In: Göhlich M, Schröer A, Weber SM (eds) Handbuch Organisationspädagogik (Organisation und Pädagogik, vol 17. Springer Fachmedien Wiesbaden, Wiesbaden, pp 17-28

Griffith J (2004) Relation of principal transformational leadership to school staff job satisfaction, staff turnover, and school performance. J Educ Adm 42(3):333-356. https://doi.org/10.1108/ 09578230410534667

Gumus S, Bellibas MS, Esen M, Gumus E (2018) A systematic review of studies on leadership models in educational research from 1980 to 2014. Educ Manag Adm Leadersh 46(1):25-48. https://doi.org/ $10.1177 / 1741143216659296$

Hallinger P, Murphy J (1985) Assessing the instructional management behavior of principals. Elem Sch J 86(2):217-247

Heissel A, Pietrek A, Flunger B, Fydrich T, Rapp MA, Heinzel S, Vansteenkiste M (2018) The validation of the German Basic Psychological Need Satisfaction and Frustration Scale in the context of mental health. Eur J Health Psychol 25(4):119-132. https://doi. org/10.1027/2512-8442/a000017

Hofmann F (2020) Authentisches und kontextsensibles Lehrerinnenund Lehrerhandeln. Das Selbst als Quelle und Ziel pädagogischen Tuns. Beltz Juventa, Weinheim

Hofmann F, Salzgeber G (2017) Qualitätsvolle pädagogische Führung im Unterricht? Pädagogische Führung 28(2):64-67

Kovjanic S, Schuh SC, Jonas K (2013) Transformational leadership and performance: an experimental investigation of the mediating effects of basic needs satisfaction and work engagement. J Occup Organ Psychol. https://doi.org/10.1111/joop.12022

Kuha J (2004) AIC and BIC. Comparisons of assumptions and performance. Sociol Methods Res 33(2):188-229. https://doi.org/10. $1177 / 0049124103262065$

Kuhl J (2001) Motivation und Persönlichkeit. Interaktionen psychischer Systeme. Hogrefe, Göttingen

Kuhl J (2010) Lehrbuch der Persönlichkeitspsychologie. Motivation, Emotion und Selbststeuerung. Hogrefe, Göttingen

Kuhl J (2013) Auswertungsmanual für den Operanten Multi-Motiv-Test OMT. Sonderpunkt-Wiss.-Verl, Münster

Kuhl J, Alsleben P (2012) Manual für die trainingsbegleitende Osnabrücker Persönlichkeitsdiagnostik. TOP. Sonderpunkt-Wiss.-Verl, Münster

Kuhl J, Scheffer D, Mikoleit B, Strehlau A (2010) Persönlichkeit und Motivation im Unternehmen. Anwendung der PSI-Theorie in Personalauswahl und -entwicklung. Kohlhammer Verlag

Kwakman K (2003) Factors affecting teachers' participation in professional learning activities. Teach Teach Educ 19(2):149-170. https://doi.org/10.1016/S0742-051X(02)00101-4

Leithwood K, Jantzi D (2005) A review of transformational school leadership research 1996-2005. Leadersh Policy Sch 4(3):177199. https://doi.org/10.1080/15700760500244769

Leithwood K, Jantzi D (2006) Transformational school leadership for large-scale reform. Effects on students, teachers, and their classroom practices. Sch Eff Sch Improv 17(2):201-227. https://doi. org/10.1080/09243450600565829

Leithwood K, Harris A, Strauss T (2010) Leading school turnaround: how successful leaders transform low-performing schools. John Wiley \& Sons

Mayrowetz D (2008) Making sense of distributed leadership. Exploring the multiple usages of the concept in the field. Educ Adm Q 44(3):424-435. https://doi.org/10.1177/0013161X07309480

McCarley TA, Peters ML, Decman JM (2016) Transformational leadership related to school climate. Educ Manag Adm Leadersh 44(2):322-342. https://doi.org/10.1177/1741143214549966

Musca SC, Kamiejski R, Nugier A, Méot A, Er-Rafiy A, Brauer M (2011) Data with hierarchical structure: impact of intraclass correlation and sample size on type-I error. Front Psychol 2:74. https:// doi.org/10.3389/fpsyg.2011.00074

Nguni S, Sleegers P, Denessen E (2006) Transformational and transactional leadership effects on teachers' job satisfaction, organizational commitment, and organizational citizenship behavior in primary schools: the Tanzanian case. Sch Eff Sch Improv 17(2):145-177. https://doi.org/10.1080/09243450600565746 
Ninković SR, Knežević Florić OČ (2018) Transformational school leadership and teacher self-efficacy as predictors of perceived collective teacher efficacy. Educ Manag Adm Leadersh 46(1):49-64. https://doi.org/10.1177/1741143216665842

Pashiardis P, Brauckmann S (2019) New Public Management in education: a call for the edupreneurial leader? Leadersh Policy Sch 18(3):485-499. https://doi.org/10.1080/15700763.2018.1475575

R Core Team (2017) R: a language and environment for statistical computing. Vienna, Austria. https://www.r-project.org/

Revelle W, Zinbarg RE (2009) Coefficients Alpha, Beta, Omega, and the glb. Psychometrika 74(1):145-154. https://doi.org/10.1007/ s11336-008-9102-Z

Rosseel Y (2012) Lavaan: an R package for structural equation modeling. J Stat Softw 48(2):1-36. http://www.jstatsoft.org/v48/i02/

Satorra A, Bentler PM (2001) A scaled difference chi-square test statistic for moment structure analysis. Psychometrika 66(4):507-514. https://doi.org/10.1007/bf02296192

Schmidt-Huber M, Dörr S, Maier GW (2014) Die Entwicklung und Validierung eines evidenzbasierten Kompetenzmodells effektiver Führung (LEaD: Leadership Effectiveness and Development). Zeitschrift Für Arbeits- Und Organisationspsychologie A\&o 58(2):80-94. https://doi.org/10.1026/0932-4089/a000138

Schratz M, Wiesner C, Kemethofer D, George AC, Rauscher E, Krenn S, Huber SG (2016) Schulleitung im Wandel: Anforderungen an eine ergebnisorientierte Führungskultur. In: Bruneforth M, Eder F, Krainer K, Schreiner C, Seel A, Spiel C (eds) Nationaler Bildungsbericht Österreich 2015, Band 2: Fokussierte Analysen bildungspolitischer Schwerpunktthemen. Leykam, Graz
Schwanenberg J, Brauckmann S, Klein ED (2020) Schulleitungen zwischen Verwaltung und Qualitätsentwicklung - wie gut gelingt die Erfüllung erweiterter Tätigkeitsfelder? Empirische Erkenntnisse aus den Forschungsprojekten Schulleitungsmonitor und SHaRP. In: Klein ED, Bremm N (eds) Unterstützung - Kooperation - Kontrolle. Zum Verhältnis von Schulaufsicht und Schulleitung in der Schulentwicklung (Educational Governance, Band 48, Bd. 48, S. 139-163). Springer VS, Wiesbaden

Shamir B, House RJ, Arthur MB (1993) The motivational effects of charismatic leadership. A self-concept based theory. Organ Sci 4(4):577-594. https://doi.org/10.1287/orsc.4.4.577

Sobanski F (2019) Warum „Grundkompetenzen absichern“? Hintergründe und Intentionen von GruKo. Schulverwaltung 7(6):162-165

Tabachnick BG, Fidell LS (2013) Using multivariate statistics. Pearson Education, Harlow

Terhart E (2013) Teacher resistance against school reform: Reflecting an inconvenient truth. Sch Leadersh Manag 33(5):486-500. https://doi.org/10.1080/13632434.2013.793494

The jamovi project (2019) Jamovi. Version 0.9. https://www.jamovi. org/

Wahrig (ed) (2000) Der kleine Wahrig Wörterbuch der deutschen Sprache. Bertelsman, Gütersloh

Wang S, Chen C-C, Dai C-L, Richardson GB (2018) A call for, and beginner's guide to, measurement invariance testing in evolutionary psychology. Evol Psychol Sci 4(2):166-178. https://doi.org/ 10.1007/s40806-017-0125-5

Yukl G (1999) An evaluation of conceptual weaknesses in transformational and charismatic leadership theories. Leadersh Q 10(2):285305. https://doi.org/10.1016/S1048-9843(99)00013-2 